\title{
A HIGHLY SENSITIVE SMALL-FOOTPRINT VOX-BASED MICRO-PIRANI GAUGE FOR IN-SITU MONITORING OF VACUUM WAFER-LEVEL PACKAGED BOLOMETERS
}

Pascal J. Newby ${ }^{l^{*}}$, Cédric Spits ${ }^{l^{* *}}$, Olivier Bernard ${ }^{1}$, Ronan Larger $^{2}$, Kevin Kornelsen $^{2}$, Paul G. Charette ${ }^{l}$, and Luc G. Fréchette ${ }^{I}$

${ }^{1}$ Institut Interdisciplinaire d'Innovation Technologique (3IT), Université de Sherbrooke, Sherbrooke, QC, Canada ${ }^{2}$ Teledyne DALSA Semiconductor Inc., 18 Boulevard de l'aéroport, Bromont, QC, Canada

\begin{abstract}
We have designed, fabricated, and tested micro-Pirani gauges, optimised for hermeticity monitoring in wafer-level packaged bolometer dies. Tests show that the gauges have a resolution of at least $1 \mathrm{mTorr}$. Furthermore, our gauges have a significantly smaller footprint than gauges previously published which achieve a similar resolution. This is due to the use of high TCR (temperature coefficient of resistance) vanadium oxide and an optimised compact design. These features make our Pirani gauges ideal for hermeticity monitoring in bolometer dies, and we have demonstrated this capacity by performing leak rate tests on wafer-level packaged dies with our gauges.
\end{abstract}

\section{INTRODUCTION}

Bolometers and infrared (IR) imaging, initially developed for military applications, are currently attracting ever-greater interest for consumer applications, as evidenced by recently released cameras, designed to be connected to a smartphone [1], [2]. A bolometer is essentially an IR-sensitive thermistor, so it must be packaged under vacuum to suppress heat loss through air, generally at a pressure on the order of 10 mTorr [3], [4].

Cost of the device is critical, and one of the key enabling technologies for reducing fabrication costs of bolometers is to replace die-level packaging with wafer-level packaging (WLP) [3], [5]. The seal must be hermetic to avoid leaks and the ensuing reduction of performance, so a method of monitoring pressure inside the bolometer throughout the lifetime of the device is extremely useful. Pirani gauges are a good choice for vacuum monitoring of bolometers: being pressure-sensitive thermistors, they are functionally similar to bolometers, so can easily be integrated in the same device using the same fabrication process.

Microfabricated Pirani gauges have been demonstrated before, but those with the required resolution have a footprint greater than $100 \times 100 \mu \mathrm{m}^{2}$. Given that another key factor for costs is the device size, it is essential that the Pirani gauge has as small a footprint as possible to avoid wasting valuable die real-estate. We have designed, fabricated, and tested micro-Pirani gauges with a resolution of at least $1 \mathrm{mTorr}$ and sizes between $22 \times 22 \mu \mathrm{m}^{2}$ and $42 \times 45 \mu \mathrm{m}^{2}$. These gauges are significantly smaller than any other previously published gauges with similar resolution. We have also used these micro-Pirani gauges to monitor hermeticity inside WLP dies.

\section{THEORY OF PIRANI GAUGES}

Micro-Pirani gauges are generally implemented using a suspended platform. On this platform there is a resistive sensing element which is heated by the Joule effect. The equilibrium temperature of the gauge depends on the heat loss, which occurs mainly via solid conduction through the structure suspending the gauge, and through the surrounding gas. The thermal conductance of the surrounding gas varies with pressure, as long as the mean free path is greater than the gap between the gauge and the substrate, so the temperature of the gauge depends on pressure. The gauge temperature is determined by measuring the electrical resistance of the suspended heating element, which varies due to its TCR (temperature coefficient of resistance). In our gauges there is a layer of vanadium oxide on the suspended platform which serves as the resistive sensing element, and since vanadium oxide has a negative TCR, resistance decreases as pressure decreases.

Research on micro-Pirani gauges initially focussed on addressing one of the main disadvantages of conventional Pirani gauges, by extending their upper pressure limit up to and beyond atmospheric pressure. This was achieved by reducing the gap between the suspended platform and the substrate [6], down to values as low as $50 \mathrm{~nm}$ [7]. More recently, it has been proposed to
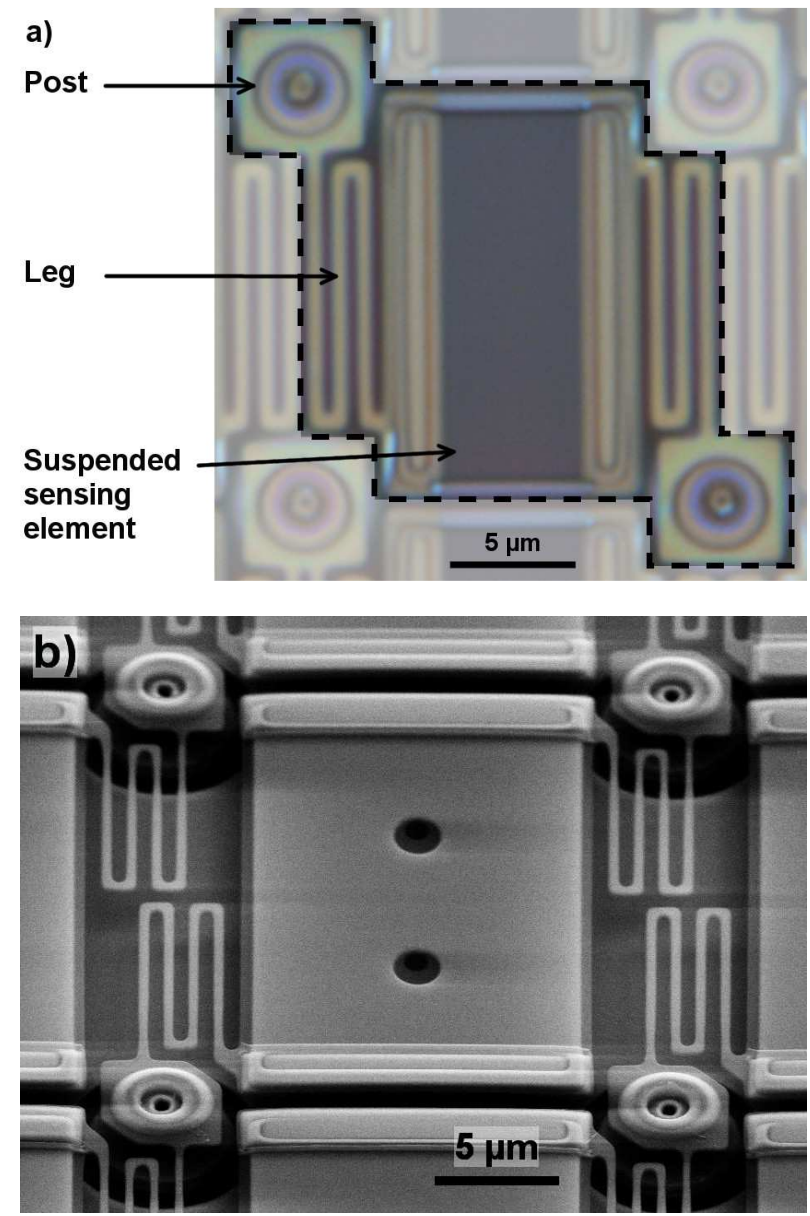

Figure 1: a) Optical microscope image showing a top-view of a $22 \times 22 \mu^{2}$ Pirani gauge. The dotted lines show the limits of the device. b) Tilted scanning electron microscope (SEM) image of a $26 \times 29 \mu \mathrm{m}^{2}$ gauge. 
use micro-Pirani gauges to monitor hermeticity of vacuumpackaged MEMS [8], so sensitivity down to the mTorr range is required.

\section{EXPERIMENTAL DETAILS}

Fabrication and design of the micro-Pirani gauges

We designed micro-Pirani gauges with four different sizes, between $22 \times 22 \mu \mathrm{m}^{2}$ and $42 \times 45 \mu \mathrm{m}^{2}$. The legs suspending the platform and sensing element are long and narrow to reduce thermal conductance of the gauges, thus increasing their resolution at lowpressure. The gauges were successfully fabricated on $200 \mathrm{~mm}$ wafers using an industrial CMOS-compatible process.

The main materials used were silicon nitride as the structural material, and high-TCR vanadium oxide as the sensing material on the suspended platform. The latter material was chosen as it is the most commonly used material for commercial micro-bolometers. Certain wafers were then wafer-level packaged by bonding them to a second wafer using a metal solder, in order to enclose the Pirani gauges in a cavity under vacuum. Fig. 1 shows images of two examples of Pirani gauges which were fabricated, with footprints of $22 \times 22 \mu \mathrm{m}^{2}$ and $26 \times 29 \mu \mathrm{m}^{2}$.

\section{Pirani gauge characterisation methods}

All electrical tests were carried out by driving the Pirani gauges with a current source and measuring their output voltages. To test the response of the gauges as a function of pressure, the gauges were placed in a vacuum prober, in which the pressure could be controlled between $10 \mu$ Torr and atmospheric pressure. Pressure was measured using an ion gauge or a capacitive diaphragm gauge, depending on the vacuum level.

Pirani gauges can also be used to measure leak rates into a WLP die, by measuring the evolution of pressure inside the die versus time. This test is usually accelerated using "bombing", in other words, by placing the die in a high pressure environment. We carried out leak rate measurements in a custom-built bombing chamber, pictured in Fig. 2, under a helium atmosphere.

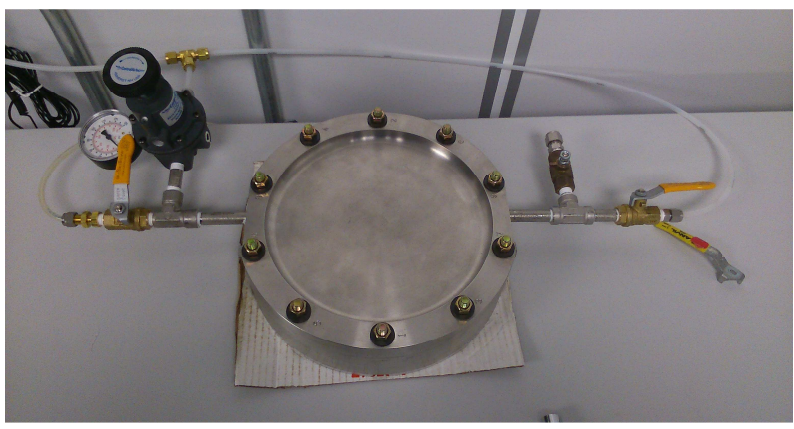

Figure 2: $200 \mathrm{~mm}$ bombing chamber used for accelerated leak rate measurements at the wafer-level with our micro-Pirani gauges.

\section{RESULTS AND DISCUSSION}

Electrical measurements of unpackaged micro-Pirani gauges

The resolution of the Pirani gauges was determined by measuring the voltage across the gauges driven at constant current, while increasing pressure in the vacuum prober. Fig. 3 shows these measurements for two different gauge sizes and we can observe that in both cases the resolution is better than 1 mTorr.

Fig. 4 shows resolution versus device footprint for the smallest Pirani gauge which we fabricated $\left(22 \times 22 \mu \mathrm{m}^{2}\right)$, compared to other Pirani gauges published in the literature [4], [6], [7], [9]-[16]. This clearly shows that a resolution of $1 \mathrm{mTorr}$ has never been achieved

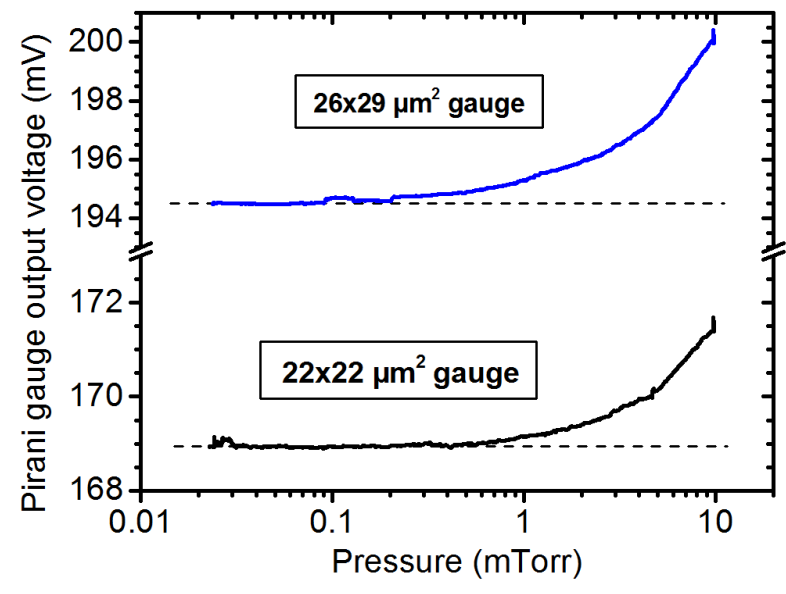

Figure 3: Measurement of the response of two Pirani gauges at constant current as a function of pressure. The $22 \times 22 \mu^{2}$ gauge has a resolution of 1 mTorr and the $26 \times 29 \mu m^{2}$ gauge has a resolution of less than 1 mTorr.

with such a small footprint; indeed the footprint of devices with a comparable resolution is at least one order of magnitude higher. Previously published work has focussed on either achieving very high resolution gauges at the cost of a larger footprint, or gauges with very small size, but which had lower resolution. The performance of our gauges was largely achieved through the use of vanadium oxide as a sensing material. Indeed, the TCR of vanadium oxide is an order of magnitude higher than that of metals, which are the most commonly-used material for sensing elements in previously published work. The footprint of the gauges was reduced by optimising their design to make them as compact as possible.

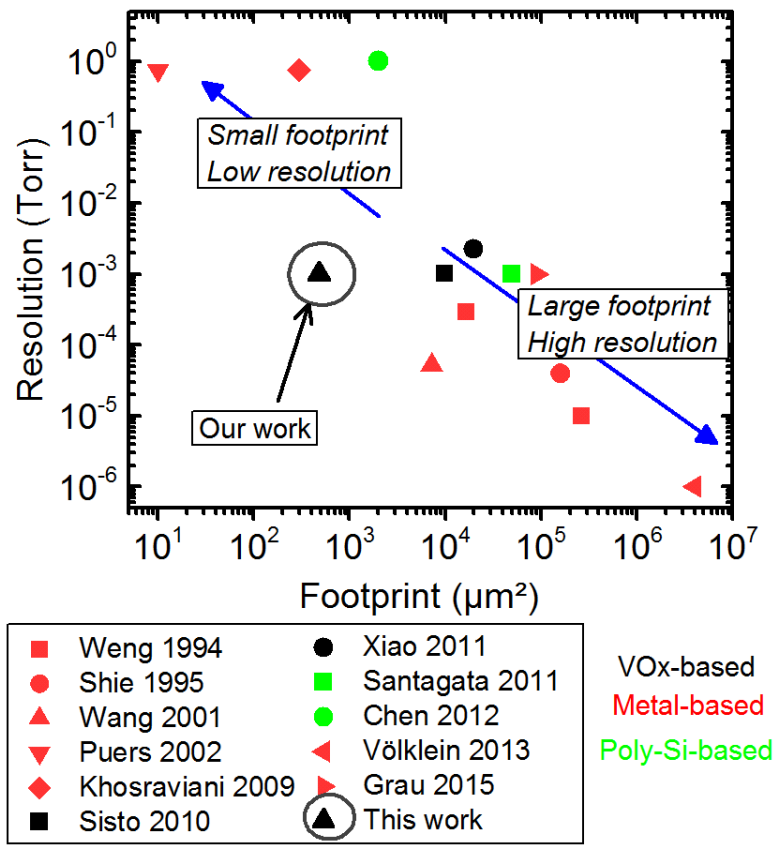

Figure 4: Resolution versus footprint for our Pirani gauge and a selection of gauges from the literature. Our gauge achieves a resolution of $1 \mathrm{mTorr}$, but with a significantly smaller footprint than other published devices. The colour of the symbols indicates the sensing material which was used. 
Fig. 5 shows the results of resistance versus current measurements performed at different constant-pressure values. These measurements demonstrate the wide operation range of the Pirani gauges, which have an upper detection limit of around 100 Torr. While this is much greater than the desired operating pressure for bolometers, this can be useful for troubleshooting hermeticity problems caused by leaks or outgassing for example.

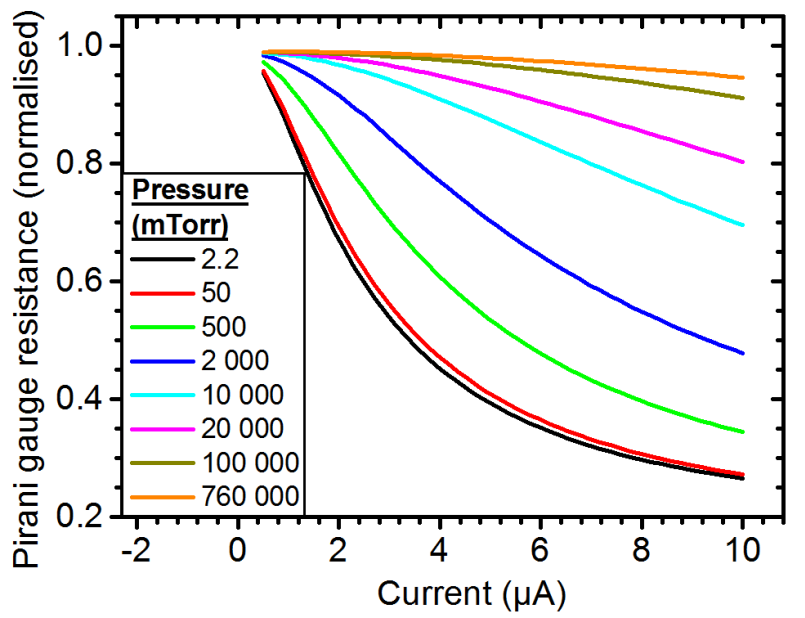

Figure 5: Pirani gauge resistance versus driving current, for different pressures. This illustrates the typical pressure-dependent behaviour of a Pirani gange: as pressure decreases, a given current will cause a greater temperature rise, leading to a greater resistance decrease (due to the negative TCR of vanadium oxide).

\section{Using the micro-Pirani gauges to monitor hermeticity of WLP} dies

Fig. 6 shows resistance versus current measured on a WLP Pirani gauge before and after bombing for 9 days under 5 atm of helium. We can see that for a given current, resistance is higher after bombing, and as explained earlier, this means that the pressure inside the die has increased, due to helium leaking through the seal. Based on the estimated pressure values, the standard leak rate is on the order of $10^{-12}$ Torr.L/s.

The maximum bombing pressure recommended to avoid creating additional leakage paths in the seal is 5 atm [17], but bombing time can easily be increased. Furthermore, the use of helium also significantly increases the sensitivity of the measurement. Leak rates are usually reported as standard leak rates, ie. the leak rate which would be measured if the die was exposed to air under a $\Delta \mathrm{P}$ of $1 \mathrm{~atm}$. To convert a standard leak rate, $\mathcal{L}$, to a leak rate, $R$, obtained with another gas at a different pressure and temperature, the following equation is used [18] :

$$
R=\mathcal{L} \sqrt{\frac{M_{\text {air }}}{M_{\text {gas }}} \frac{T_{\text {gas }}}{T_{293 K}}} \frac{P_{\text {ext }}-P_{\text {die }}}{P_{\text {atm }}}
$$

where $M_{\text {air }}$ and $M_{\text {gas }}$ are the atomic weights of air and the gas used, $T_{g a s}$ is the temperature of the gas, $P_{e x t}$ is the external pressure, $P_{\text {die }}$ the pressure inside the die, and $P_{a t m}$ the standard atmospheric pressure. Therefore, by using helium at $5 \mathrm{~atm}$, the leak test is accelerated by a factor of 13 . Furthermore, the sensitivity of the Pirani gauges is improved, given that the thermal conductivity of helium is six times higher than that of air. Therefore, by carrying out bombing during one month, we estimate that we will be able to measure leak rates as low as $10^{-15}$ Torr.L/s, which compares favourably with RGA (residual gas analysis) measurements.

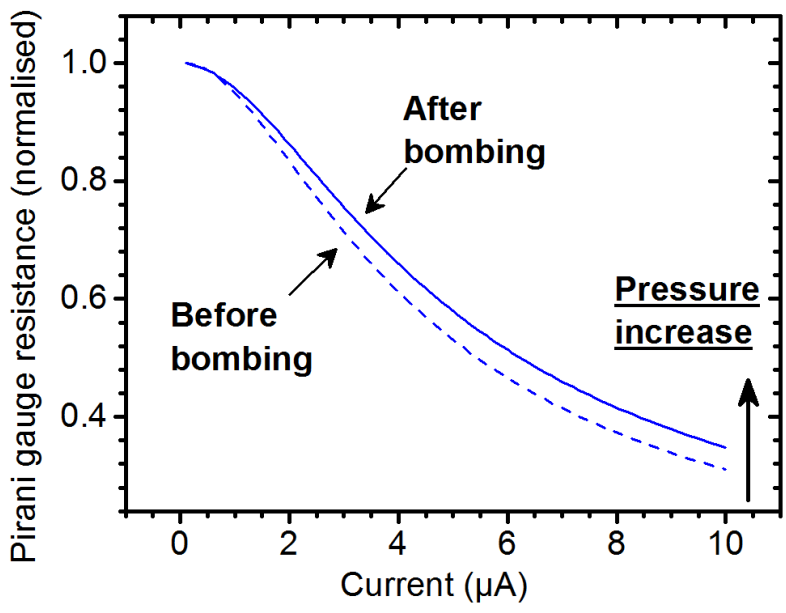

Figure 6: Resistance versus current for a WLP Pirani gauge, before and after bombing during 9 days at 5 atm of helium. After bombing, the curve has shifted upwards due to the pressure increase inside the die caused by helium leaking in.

\section{CONCLUSIONS}

We have successfully designed and fabricated micro-Pirani gauges on $200 \mathrm{~mm}$ wafers, using a CMOS-compatible industrial process. We also packaged devices under vacuum, using waferlevel packaging. Electrical testing shows that the gauges have a resolution of at least $1 \mathrm{mT}$ Torr, which has never been achieved previously with such a small footprint. This performance was obtained thanks to the use of high-TCR vanadium oxide and the use of this material also makes these Pirani gauges an ideal choice for in-situ monitoring of WLP bolometers. We have also used these gauges to carry out leak rate measurements to evaluate the hermeticity of WLP dies. Pirani gauges are usually calibrated as a function of pressure before vacuum packaging, or one die is calibrated and used for all other dies from the wafer [4]. Both approaches are time-consuming, and even with a well-controlled process, the latter approach increases measurement uncertainty [4]. Therefore, we are currently developing methods for measuring pressure within a packaged die which do not require prior calibration versus pressure.

\section{ACKNOWLEDGEMENTS}

The authors wish to thank NSERC, Teledyne DALSA Semiconductor, PROMPT-Québec, and MITACS for funding of this project, and are grateful for the invaluable assistance of the staff of Teledyne DALSA Semiconductor involved in the fabrication of these devices. Fabrication was partly carried out at C2MI. Travel support has been generously provided by the Transducer Research Foundation.

\section{REFERENCES}

[1] FLIR Systems, Inc., http://www.flir.ca/flirone/, page visited April $3^{\text {rd }}, 2016$.

[2] Seek Thermal Inc., http:/www.thermal.com/products/ compact, page visited April $3^{\text {rd }}, 2016$.

[3] F. Forsberg, N. Roxhed, A.C. Fischer, B. Samel, P. Ericsson, N. Hoivik, A. Lapadatu, M. Bring, G. Kittilsland, G. Stemme, and F. Niklaus, "Very Large Scale Heterogeneous Integration (VLSHI) And Wafer-level Vacuum Packaging For Infrared Bolometer Focal Plane Arrays", Infrared Physics \& 
Technology, 60, 251 (2013).

[4] M.M. Sisto, S. García-Blanco, L. Le Noc, B. Tremblay, Y. Desroches, J.-S. Caron, F. Provencal, and F. Picard, "Pressure Sensing In Vacuum Hermetic Micropackaging For MOEMSMEMS", Journal of Micro/Nanolithography, MEMS and MOEMS, 9, 041109 (2010).

[5] P. Schweikert, A. Sharpe, G.A. Carlson, A. Matson, S. Vilander, B. Zahuta, and R.M. Goeden, "Wafer Level Packaging Of Microbolometer Vacuum Package Assemblies," US patent application 20140186999 A1, publication date July 3, 2014.

[6] R. Puers, S. Reyntjens, and D. De Bruyker, "The NanoPiranian Extremely Miniaturized Pressure Sensor Fabricated By Focused Ion Beam Rapid Prototyping", Sensors and Actuators A, 97-98, 208 (2002).

[7] K. Khosraviani and A.M. Leung, "The Nanogap Pirani-a Pressure Sensor With Superior Linearity In An Atmospheric Pressure Range", Journal of Micromechanics and Microengineering, 19, 045007 (2009).

[8] E.S. Topalli, K. Topalli, S.E. Alper, T. Serin, and T. Akin, "Pirani Vacuum Gauges Using Silicon-on-glass And Dissolved-wafer Processes For The Characterization Of MEMS Vacuum Packaging”, IEEE Sensors Journal, 9, 263 (2009).

[9] P.K. Weng and J.-S. Shie, "Micro-Pirani Vacuum Gauge", Review of Scientific Instruments, 65, 492 (1994).

[10] J.-S. Shie, B.C.S. Chou, and Y.-M. Chen, "High Performance Pirani Vacuum Gauge", Journal of Vacuum Science \& Technology A, 13, 2972 (1995).

[11] S.N. Wang, K. Mizuno, M. Fujiyoshi, H. Funabashi, and J. Sakata, "Thermal Micropressure Sensor For Pressure Monitoring In A Minute Package", Journal of Vacuum Science \& Technology A, 19, 353 (2001).

[12] B. Xiao, T. Dong, E. Halvorsen, Z. Yang, Y. Zhang, N. Hoivik, D. Gu, N.M. Tran, and H. Jakobsen, "Integrated Micro Pirani Gauge Based Hermetical Package Monitoring For Uncooled VOx Bolometer FPAs", Microsystem Technologies, 17, 115 (2011).

[13] F. Santagata, J.F. Creemer, E. Iervolino, L. Mele, A.W. van Herwaarden, and P.M. Sarro, "A Tube-Shaped Buried Pirani Gauge For Low Detection Limit With Small Footprint", Journal of Microelectromechanical Systems, 20, 676 (2011).

[14] C.-N. Chen, "Characterization Of Gas Conductance Of A Thermal Device With A V-Groove Cavity", IEEE Electron Device Letters, 33, 275 (2012).

[15] F. Völklein, M. Grau, A. Meier, G. Hemer, L. Breuer, and P. Woias, "Optimized MEMS Pirani Sensor With Increased Pressure Measurement Sensitivity In The Fine And High Vacuum Regime", Journal of Vacuum Science \& Technology A, 31, 061604 (2013).

[16] M. Grau, F. Völklein, A. Meier, C. Kunz, I. Kaufmann, and P. Woias, "Optimized MEMS Pirani Sensor With Increased Pressure Measurement Sensitivity In The Fine And Rough Vacuum Regimes", Journal of Vacuum Science \& Technology A, 33, 021601 (2015).

[17] S. Costello, M.P.Y. Desmulliez, and S. McCracken, "Review Of Test Methods Used For The Measurement Of Hermeticity In Packages Containing Small Cavities", IEEE Transactions on Components, Packaging and Manufacturing Technology, 2, 430 (2012)

[18] D.A. Howl and C.A. Mann, “The Back-pressurising Technique Of Leak-testing", Vacuum, 15, 347 (1965).

\section{CONTACT}

*Pascal J. Newby, Pascal.Newby@,USherbrooke.ca

**Cédric Spits, Cedric.Spits@usherbrooke.ca 